\title{
Approximate Bayesian Estimation for Parameters of Simple Linear Bivariate Truncated T Regression Model
}

\section{Elham Abdulkreem Hussain $^{1 *}$; Haifa Abduljwaad Saied ${ }^{2}$}

\footnotetext{
${ }^{1}$ Department of Technical Product plant, Technical Agriculture College-Mosul,Northern Technical University

${ }^{2}$ Department of Statistic and Informatics, College of Computer Sciences and Mathematics, Mosul University
}

$$
\text { Email: }{ }^{1 *} \text { dr.elham@ntu.edu.iq, }{ }^{2} \text { haeifa965@gmail.com }
$$

(Received September 03, 2018; Accepted December 03, 2019; Available online March 01, 2020)

DOI: 10.33899/edusj.2020.164372, (C) 2020, College of Education for Pure Science, University of Mosul.

This is an open access article under the CC BY 4.0 license (http://creativecommons.org/licenses/by/4.0/).

\begin{abstract}
:
In this paper, it is obtained to approximate estimation for parameters of the two-tailed truncated regression model by Lindely approach. The prior non- informative was used for the regression parameters matrix when the variance matrix is known and the two truncated points are also known. Under the quadratic loss function, the estimates approximations of the regression parameters matrix around zero up to third order moments are obtained.
\end{abstract}

Keywords: truncated regression, Lindely's method, $\mathrm{t}$ regression model

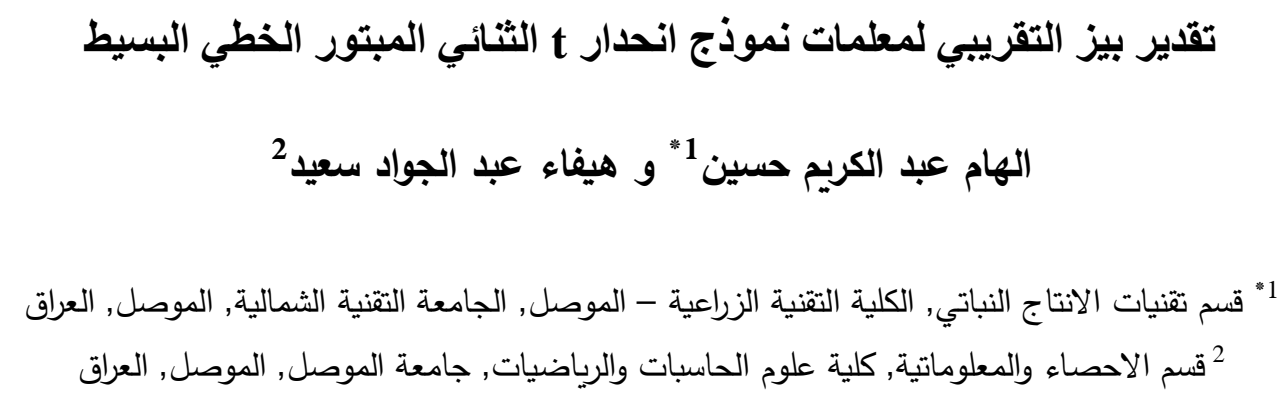

المستخلص

تم في هذا البحث الحصول على التقدير التقريبي لمعلمات نموذج انحدار t الثنائي المبتور من طرفين بطريقة لندلي ، وتم استخدام معلومات سابقة غير خبرية حول مصفوفة معلمات الانحدار عندما تكون مصفوفة التباين معلومة ونقطتي البتر لمات

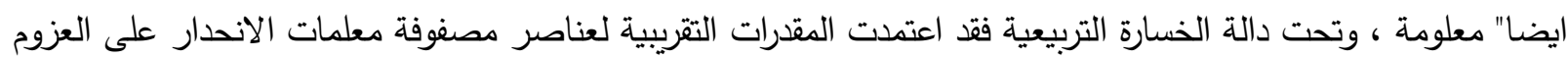
حول الصفر لغاية المرتبة الثالثة . الكلمات المفتاحية : الانحدار المبتور , طريقة لندلي , نموذج انحدار t 
1

تناول الباحثون خلال السنين الماضية موضوع تحليل الانحدار المبتور متعدد المتغيرات لما لهذا الموضوع من اهمية لدراسة العديد من الظواهر الاقتصادية والاجتماعية والطبيعية وغيرها ، فقد قدم [1] [Frenandes and Steel بحثاً تتاول فيه تقدير معلمات نموذج الانحدار المبتور متعدد المتغيرات باستخدام الاسلوب الكلاسيكي واسلوب بيز مع افتراض ان الخطأ يتوزع توزيع t ـ قدم [Yan [2 بحثاً تناول فيه تقدير المعلمة م لنموذج انحدار خطي حسب طريقة المربعـات الصغـرى،

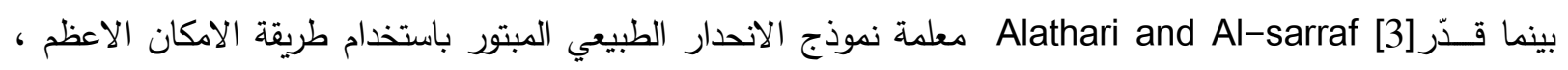
وعرض [Massuia et. al. [4 التحليل التشخيصي لنموذج الانحدار الخطي مع بتر متغير الاستجابة وان الخطأ فيه يتبع لئع توزيع t ، و قدموا خلال البحث تقدير المعلمات بطريقة تعظيم التوقـع Maximization Expected • بعض Ho. et. al. [5 مض النتائج عن متعدد المتغيرات لتوزيع t المبتور ، حيث كانت النتائج التي قدمها تتعلق بحساب الوسط والتباين عن طريق احتساب العزوم من الرتبة الاولى والثانية عندما يكون هناك بتر من الطرفين ، وقدم حالة خاصة ايضا" عنما يكون البتر من جهة اليمين مع اعطاء بعض الامثلة والتطبيقات . ان لتوزيع t استخدامات كثيرة في التطبيقات العملية كما في تطبيقات النظرية الاحصائية ـتزايد الاهتمام في الـ (30 20 ) سنة الماضية بتوزيع t المتعدد المتغيرات [6]، فبالإضافة الى الندذجة الاحصائية التقليدية فقد أُستخدم التوزيع في

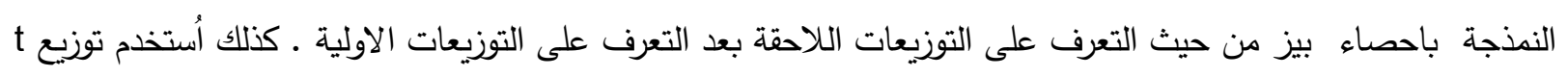

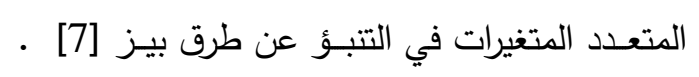

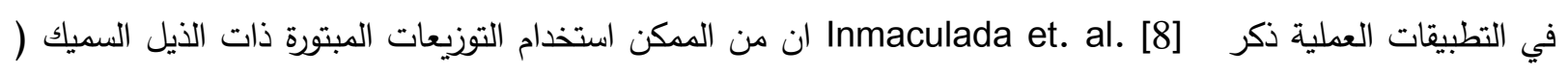
ومنها توزيع t ) في التطبيقات المالية والفيزيائية والهيدرولوكية (علم المياه) والهندسية وتطبيقات اخرى مثل الزلازل الكبيرة

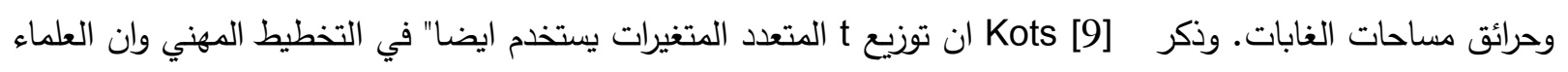

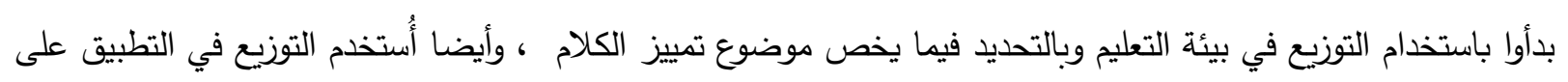

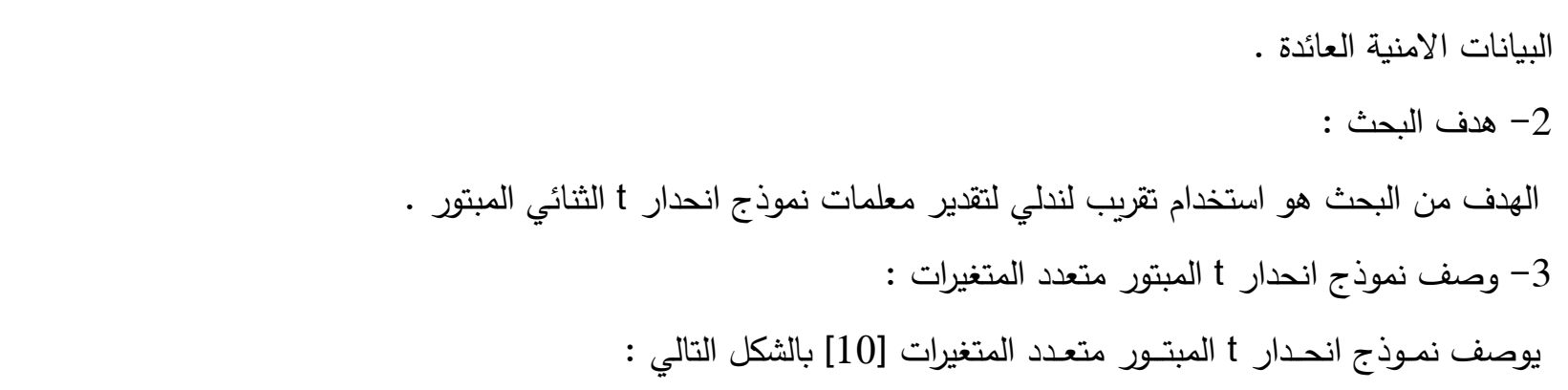

$\underline{y_{i}}=\beta \underline{x}_{i}+\underline{u}_{i}$

$\underline{y_{i}}=\left(\begin{array}{ll}y_{1 i} & y_{2 i}\end{array}\right)^{\prime}$

: حيث ان

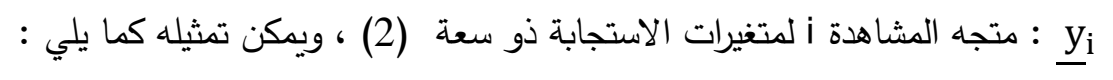

: متجه للمشاهدة i للمتغيرات التوضيحية ويأخذ الثكل التالي 
$\underline{x_{i}}=\left(\begin{array}{c}1 \\ x_{i 1}\end{array}\right)$

$\boldsymbol{\beta}=\left[\begin{array}{ll}\beta_{01} & \beta_{11} \\ \beta_{02} & \beta_{12}\end{array}\right]$

اما المصفوفة م فهي تمثل مصفوفة معلمات الانحدار ذات سعة 2*2 وتأخذ الثكل الاتي :

$\underline{u}_{i}=\left(\underline{u}_{1 i} \underline{u}_{2 i}\right)^{\prime}$

: متجه الخطأ العشوائي للمشاهدة i ذو سعة (k ويكون كالثكل التالي :

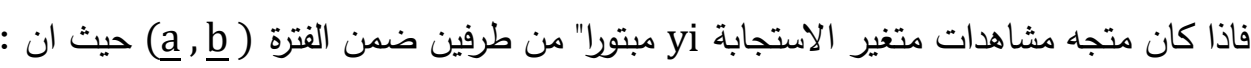

$\underline{a}=\left(\begin{array}{ll}a_{1} & a_{2}\end{array}\right)^{\prime}$

$\underline{b}=\left(\begin{array}{ll}b_{1} & b_{2}\end{array}\right)^{\prime}$

وكان yi يتبع توزيع t متعدد المتغيرات المبتور من طرفين ضمن الفترة (

$y_{i} \mid \beta, \Sigma \sim t_{2 T_{(\underline{a}, \underline{b})}}\left(\beta x_{i}, \Sigma, v\right)$

وباستخدام النظرية التي ذكرها [11 مelly التي تتص على ان الدالة الخطية في متغير طبيعي مبتور مضافا" اليه ثابت يتبع التوزيع الطبيعي المبتور وباستخدام التوزيعات المختلة فأن :

$y_{i} \mid \beta, \Sigma, \mathrm{T} \sim N_{T_{(\underline{a}, \underline{b})}}\left(\beta x_{i}, \tau \Sigma\right)$

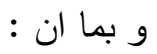

$u_{i}=y_{i}-\beta x_{i}$

$u_{i} \mid \Sigma \sim N_{T_{(\underline{a}, \underline{b})}}(o, \tau \Sigma)$

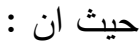

$a_{1}=a-\beta x_{i}$

$b_{1}=b-\beta x_{i}$

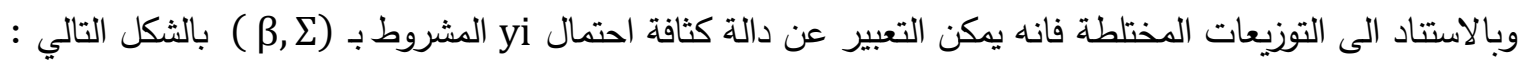

$f\left(y_{i} \mid \beta, \Sigma\right)=\frac{\int_{0}^{\infty} f\left(y_{i} \mid \beta, \Sigma, \tau\right) f(\tau) d \tau}{F(\underline{b})-F(\underline{a})}=\frac{\int_{0}^{\infty} f\left(y_{i} \mid \beta, \Sigma, \tau\right) f(\tau) d \tau}{\int_{0}^{\infty} \int_{a}^{b} f\left(y_{i} \mid \beta, \Sigma, \tau\right) f(\tau) d y_{i} d \tau}$

ان دالة الامكان المشروطة بـ $\tau$ هي : (2)

$L(\beta \mid \tau)=\left[\frac{1}{F^{*}(b \mid \tau)-F^{*}(a \mid \tau)}\right]^{n} \cdot \frac{e^{-\frac{1}{2 \tau} \Sigma\left(y_{i}-\beta x_{i}\right)^{\prime} \Sigma^{-1}\left(y_{i}-\beta x_{i}\right)}}{(2 \pi \tau)^{n / 2}|\Sigma|^{n / 2}}$ 
$F^{*}(b \mid \tau)-F^{*}(a \mid \tau)=\int_{\underline{a}}^{\underline{b}} \frac{e^{-\frac{1}{2 \tau}\left(y_{i}-\beta x_{i}\right)^{\prime} \Sigma^{-1}\left(y_{i}-\beta x_{i}\right)}}{2 \pi \tau|\Sigma|^{\frac{1}{2}}} d y_{i}$

وبافتراض توفير معلومات اولية غير خبرية حول مصفوفة معلمات الانحدار م المشروطة بـ $\tau$ هي [12] :

$p(\beta, \Sigma \mid \tau) \propto$ constant $c=(\Sigma)^{-\frac{p+1}{2}}$

وبعد دمج معلومات العينة مع المعلومات المسبقة حول مصفوفة معلمات الاتحدار م و باستخدام نظرية بيز يكون التوزيع اللاحق لـ $\beta$ المشروط بـ $\tau$ :

$p(\beta \mid \tau$, data $)=\frac{L(\beta \mid \tau) p(\beta \mid \tau)}{\int_{\beta} L(\beta \mid \tau) p(\beta \mid \tau) d \underline{\beta}}$

4

تحت دالة الخسارة التربيعية [13] يكون مقدر بيز لمصفوفة المعلمات ح|م هو التوقع الرياضي للتوزيع البعدي لمصفوفة المعلمات $\beta$ المشروط بـ $\tau$ الذي سبق تعريفه في المعادلة (3) اعلاه اي ان :

$\hat{\beta}_{B} \mid \tau=\frac{\int \beta L(\beta \mid \tau, \text { data }) p(\beta \mid \tau) d \beta}{\int L(\beta \mid \tau, \text { data }) p(\beta \mid \tau) d \beta}$

من الصعب جدا ايجاد قيمة التكامل اعلاه تحليليا" لذلك لجئنا الى تقريب لندلي لايجاد مقدر بيز التقريبي [14] وكالاتي : $u(\beta \mid \tau)=\operatorname{vec}(\beta \mid \tau)$

$$
=\left(\begin{array}{llll}
\beta_{10} & \beta_{20} & \beta_{11} & \beta_{21}
\end{array}\right)^{\prime}
$$

$E(u \mid \beta, \tau) \cong u(\beta \mid \tau)$

وتقريب لندلي للتوقع اللاحق لـ u( u يكون بالثكل التالي :

$$
\begin{aligned}
& +\frac{1}{2} \sum_{i=1}^{4} \sum_{i=1}^{4}\left[\left(u_{i j} \mid \tau+2\left(u_{i} \mid \tau\right)\left(\rho_{j} \mid \tau\right)\right]\left(V_{i j} \mid \tau\right)\right. \\
& \left.+\frac{1}{2} \sum_{i=1}^{4} \sum_{i=1}^{4} \sum_{i=1}^{4} \sum_{i=1}^{4}\left[\left(L_{i j k} \mid \tau\right)\left(V_{i j} \mid \tau\right)\left(V_{k l} \mid \tau\right) u_{l} \mid \tau\right)\right]
\end{aligned}
$$

ويتم ايجاد مكونات المعادلة (9) كالاتي :

أر : تمثل المشتقة الجزئية الاولى للوغاريتم الطبيعي للتوزيع الاولي لمصفوفة معلمات الانحدار م نسبة الى كل معلمة $\rho_{10}=\frac{\partial \rho}{\partial \beta_{10}}=\frac{\partial \ln p(\beta \mid \tau)}{\partial \beta_{10}} \quad, \quad \rho_{11}=\frac{\partial \rho}{\partial \beta_{11}}=\frac{\partial \ln p(\beta \mid \tau)}{\partial \beta_{11}}$ من معلمات المصفوفة $\beta$ وكالاتي : 
$\rho_{20}=\frac{\partial \rho}{\partial \beta_{20}}=\frac{\partial \ln p(\beta \mid \tau)}{\partial \beta_{10}} \quad, \quad \rho_{21}=\frac{\partial \rho}{\partial \beta_{21}}=\frac{\partial \ln p(\beta \mid \tau)}{\partial \beta_{10}}$

علماً ان جميع المشتقات الجزئية اعلاه تساوي الصفر ·

سوف يتم تقدير اربع معلمات لنموذج الانحدار وهي (

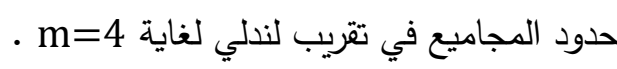
وحيث ان : 20 - n

$f\left(y_{i} \mid B, \Sigma, \tau\right)=\frac{1}{F(b \mid \tau)-F(a \mid \tau)(2 \pi \tau)(\Sigma)^{1 / 2}} e^{-\frac{\left(y_{i}-\beta X\right)^{\prime} \Sigma^{-1}\left(y_{i}-\beta X\right)}{2 \tau}}$

$F(b \mid \tau)-F(a \mid \tau)=\alpha=\int_{a}^{b} \frac{1}{(2 \pi \tau)(\Sigma)^{1 / 2}} e^{-\frac{\sum\left(y_{i}-\beta X\right)^{\prime} \Sigma^{-1}\left(y_{i}-\beta X\right)}{2 \tau}} d y_{i}$

$L(B \mid \Sigma, \tau)=\frac{1}{\alpha^{*}(2 \pi \tau)^{n}(\Sigma)^{n / 2}} e^{-\frac{\left(y_{i}-\beta X\right)^{\prime} \Sigma^{-1}\left(y_{i}-\beta X\right)}{2 \top}}$

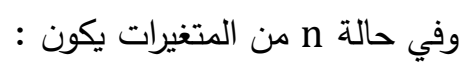

$\alpha^{*}=\int_{a}^{b} \frac{1}{(2 \pi \tau)^{n}(\Sigma)^{n / 2}} e^{-\frac{\sum_{i=1}\left(y_{i}-\beta X\right)^{\prime} \Sigma^{-1}\left(y_{i}-\beta X\right)}{2 \tau}} d y$

$Q_{i}=\left(y_{i}-\beta X\right)^{\prime} \Sigma^{-1}\left(y_{i}-\beta X\right)$

$\Sigma^{-1}=\left[\begin{array}{ll}\sigma_{11}^{*} & \sigma_{12}^{*} \\ \sigma_{21}^{*} & \sigma_{22}^{*}\end{array}\right]=\left[\begin{array}{c}\Sigma_{1}^{*} \\ \Sigma_{2}^{*}\end{array}\right]$

$\Sigma_{1}^{*}=\left[\begin{array}{ll}\sigma_{11}^{*} & \sigma_{12}^{*}\end{array}\right], \quad \Sigma_{2}^{*}=\left[\begin{array}{ll}\sigma_{21}^{*} & \sigma_{22}^{*}\end{array}\right]$

$\frac{\partial Q_{i}}{\partial \beta_{10}}=-2 \Sigma_{1}^{*}\left(y_{i}-\beta x_{i}\right) ; \frac{\partial Q_{i}}{\partial \beta_{10}}=-2 \Sigma_{1}^{*} x_{i}\left(y_{i}-\beta x_{i}\right)$

$\frac{\partial Q_{i}}{\partial \beta_{20}}=-2 \Sigma_{2}^{*}\left(y_{i}-\beta x_{i}\right) ; \frac{\partial Q_{i}}{\partial \beta_{21}}=-2 \Sigma_{2}^{*} x_{i}\left(y_{i}-\beta x_{i}\right)$

$L_{1}=\frac{\partial \operatorname{lnl}}{\partial \beta_{10}}=\frac{1}{\tau}\left[n \bar{y}-\sum_{i=1}^{n} E\left(c_{i} \mid \tau\right)\right]$

$L_{2}=\frac{\partial \ln l}{\partial \beta_{11}}=\frac{\sum_{i=1}^{n} x_{i}}{\tau}\left[y_{i}-E\left(c_{i} \mid \tau\right)\right]$ 


$$
\begin{aligned}
& L_{3}=\frac{\partial \ln l}{\partial \beta_{20}}=\frac{1}{\tau}\left[n \bar{y}-\sum_{i=1}^{n} E\left(c_{1} \mid \tau\right)\right] \\
& L_{4}=\frac{\partial \ln l}{\partial \beta_{21}}=\frac{\sum_{i=1}^{n} x_{i}}{\tau}\left[y_{i}-E\left(c_{1} \mid \tau\right)\right] \\
& c_{i}=\Sigma_{1}^{*} y_{i} ; c_{1}=\Sigma_{2}^{*} y_{i}
\end{aligned}
$$

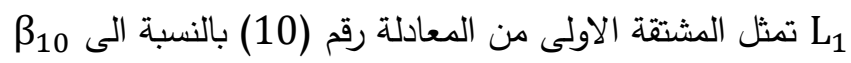

المثل المشتقة الاولى من المعادلة رقم (10) بالنسبة الى L $_{2}$

政

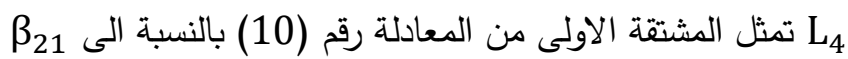

$$
\begin{aligned}
& L_{11}=\frac{\partial L_{1}}{\partial \beta_{10}}=-\frac{1}{\tau^{2}} \sum_{i=1}\left[E\left(c_{i}^{2} \mid \tau\right)-\left(E\left(c_{i} \mid \tau\right)\right)^{2}\right] \\
& L_{12}=\frac{\partial L_{1}}{\partial \beta_{11}}=-\frac{\sum_{i=1}^{n} x_{i}}{\tau^{2}}\left[E\left(c_{i}^{2} \mid \tau\right)-\left(E\left(c_{i} \mid \tau\right)\right)^{2}\right] \\
& L_{13}=\frac{\partial L_{1}}{\partial \beta_{20}}=-\frac{1}{\tau^{2}} \sum_{i=1}\left[E\left(c_{i} c_{1} \mid \tau\right)-E\left(c_{i} \mid \tau\right) E\left(c_{1} \mid \tau\right)\right] \\
& L_{14}=\frac{\partial L_{1}}{\partial \beta_{11}}=-\frac{\sum_{i=1}^{n} x_{i}}{\tau^{2}}\left[E\left(c_{i} c_{1} \mid \tau\right)-E\left(c_{i} \mid \tau\right) E\left(c_{1} \mid \tau\right)\right] \\
& L_{21}=L_{12} \\
& L_{22}=\frac{\partial L_{2}}{\partial \beta_{11}}=-\frac{\sum_{i=1}^{n} x_{i}^{2}}{\tau^{2}}\left[E\left(c_{i}^{2} \mid \tau\right)-\left(E\left(c_{i} \mid \tau\right)\right)^{2}\right] \\
& L_{23}=\frac{\partial L_{2}}{\partial \beta_{20}}=-\frac{\sum_{i=1}^{n} x_{i}}{\tau^{2}}\left[E\left(c_{i} c_{1} \mid \tau\right)-E\left(c_{i} \mid \tau\right) E\left(c_{1} \mid \tau\right)\right] \\
& L_{33}=\frac{\partial L_{3}}{\partial \beta_{20}}=-\frac{1}{\tau^{2}} \sum_{i=1}\left[E\left(c_{1}^{2} \mid \tau\right)-\left(E\left(c_{1} \mid \tau\right)\right)^{2}\right] \\
& L_{24}=\frac{\partial L_{2}}{\partial \beta_{11}} \\
& L_{31}=\frac{\sum_{i=1}^{n} x_{i}{ }^{2}}{\tau^{2}}\left[E\left(c_{i} c_{1} \mid \tau\right) E\left(c_{1} \mid \tau\right)-\sum_{1}^{*} \beta x_{i} E\left(c_{i} \mid \tau\right)\right] \\
& L_{32}=L_{23} \beta x_{i} E\left(c_{i} \mid \tau\right)
\end{aligned}
$$




$$
\begin{aligned}
& L_{34}=\frac{\partial L_{3}}{\partial \beta_{11}}=-\frac{\sum_{i=1}^{n} x_{i}}{\tau^{2}}\left[E\left(c_{1}^{2} \mid \tau\right)-\left(E\left(c_{1} \mid \tau\right)\right)^{2}\right] \\
& L_{44}=\frac{\partial L_{3}}{\partial \beta_{11}}=-\frac{\sum_{i=1}^{n} x_{i}^{2}}{\tau^{2}}\left[E\left(c_{1}^{2} \mid \tau\right)-\left(E\left(c_{1} \mid \tau\right)\right)^{2}\right]
\end{aligned}
$$

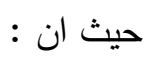

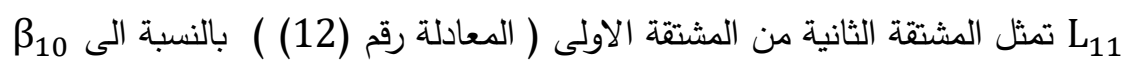

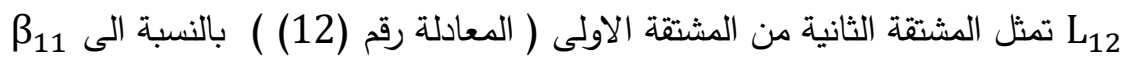

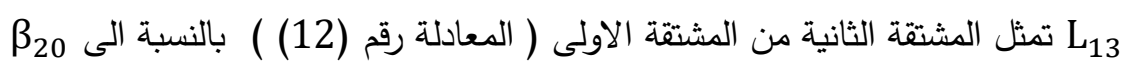

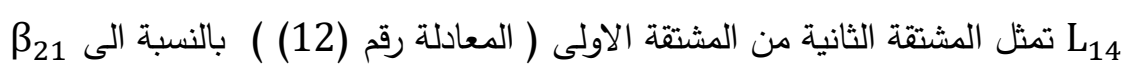
وكذا بالنسبة الى المشتقات الباقية . وبهذا تصبح مصفوفة المشتقات الثانية المتماتلة كما يلي :

$L^{2}=\left[\begin{array}{cccc}L_{11} & L_{12} & L_{13} & L_{14} \\ \vdots & L_{22} & L_{23} & L_{24} \\ \vdots & \cdots & L_{33} & L_{34} \\ \vdots & \cdots & \cdots & L_{44}\end{array}\right]$

اما العناصر Lij : تمثل العنصر (ij) من معكوس المصفوفة (L2)

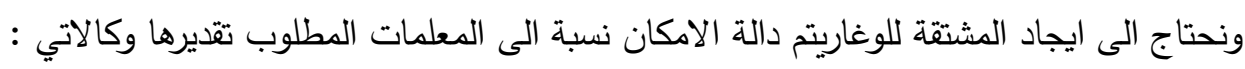

$$
\begin{aligned}
L_{111}=\frac{\partial L_{11}}{\partial \beta_{10}}= & -\frac{1}{\tau^{3}} \sum_{i=1}\left[E\left(c_{i}^{3} \mid \tau\right)-2 \Sigma_{1}^{*} \beta x_{i} E\left(c_{i}^{2} \mid \tau\right)-E\left(c_{i} \mid \tau\right) E\left(c_{i}^{2} \mid \tau\right)+\Sigma_{1}^{*} \beta x_{i}\left(E\left(c_{i} \mid \tau\right)\right)^{2}\right. \\
& \left.+2\left(E\left(c_{i} \mid \tau\right)\right)^{3}\right] \\
L_{112}=\frac{\partial L_{11}}{\partial \beta_{11}}= & -\frac{\sum_{i=1} x_{i}}{\tau^{3}}\left[E\left(c_{i}^{3} \mid \tau\right)-2 \Sigma_{1}^{*} \beta x_{i} E\left(c_{i}^{2} \mid \tau\right)-E\left(c_{i} \mid \tau\right) E\left(c_{i}^{2} \mid \tau\right)+\Sigma_{1}^{*} \beta x_{i}\left(E\left(c_{i} \mid \tau\right)\right)^{2}\right. \\
& \left.+2\left(E\left(c_{i} \mid \tau\right)\right)^{3}\right] \\
L_{113}=\frac{\partial L_{11}}{\partial \beta_{20}}= & -\frac{1}{\tau^{3}} \sum_{i=1}\left[E\left(c_{i}^{2} \mid \tau\right) E\left(c_{1} \mid \tau\right)+3 \Sigma_{1}^{*} \beta x_{i} E\left(c_{i} \mid \tau\right) E\left(c_{1} \mid \tau\right)-E\left(c_{1} \mid \tau\right) E\left(c_{i}^{2} \mid \tau\right)\right. \\
& -2 E\left(c_{i} \mid \tau\right) E\left(c_{i} c_{1} \mid \tau\right)+\Sigma_{1}^{*} \beta x_{i} E\left(c_{i} c_{1} \mid \tau\right) \\
& \left.+2 E\left(c_{1} \mid \tau\right)\left(E\left(c_{i} \mid \tau\right)\right)^{2}\right]
\end{aligned}
$$




$$
\begin{aligned}
& L_{114}=\frac{\partial L_{11}}{\partial \beta_{21}}=-\frac{\sum_{i=1} x_{i}}{\tau^{3}}\left[E\left(c_{i}^{2} \mid \tau\right) E\left(c_{1} \mid \tau\right)+3 \Sigma_{1}^{*} \beta x_{i} E\left(c_{i} \mid \tau\right) E\left(c_{1} \mid \tau\right)-E\left(c_{1} \mid \tau\right) E\left(c_{i}^{2} \mid \tau\right)\right. \\
& -2 E\left(c_{i} \mid \tau\right) E\left(c_{i} c_{1} \mid \tau\right)+\Sigma_{1}^{*} \beta x_{i} E\left(c_{i} c_{1} \mid \tau\right) \\
& \left.+2 E\left(c_{1} \mid \tau\right)\left(E\left(c_{i} \mid \tau\right)\right)^{2}\right] \\
& L_{121}=\frac{\partial L_{12}}{\partial \beta_{10}}=-\frac{\sum_{i=1} x_{i}}{\tau^{3}}\left[E\left(c_{i}^{3} \mid \tau\right)-2 \Sigma_{1}^{*} \beta x_{i} E\left(c_{i}^{2} \mid \tau\right)-E\left(c_{i} \mid \tau\right) E\left(c_{i}^{2} \mid \tau\right)\right. \\
& +2 \Sigma_{1}^{*} \beta x_{i}\left(E\left(c_{i} \mid \tau\right)\right)^{2} \\
& \left.-2 E\left(c_{i} \mid \tau\right)^{3}\right] \\
& L_{122}=\frac{\partial L_{12}}{\partial \beta_{11}}=-\frac{\sum_{i=1} x_{i}^{2}}{\tau^{3}}\left[E\left(c_{i}^{3} \mid \tau\right)-2 \Sigma_{1}^{*} \beta x_{i} E\left(c_{i}^{2} \mid \tau\right)-E\left(c_{i} \mid \tau\right) E\left(c_{i}^{2} \mid \tau\right)\right. \\
& \left.+2 \Sigma_{1}^{*} \beta x_{i}\left(E\left(c_{i} \mid \tau\right)\right)^{2}-2 E\left(c_{i} \mid \tau\right)^{3}\right] \\
& L_{123}=\frac{\partial L_{12}}{\partial \beta_{20}}=-\frac{\sum_{i=1} x_{i}}{\tau^{3}}\left[E\left(c_{i}^{2} c_{1} \mid \tau\right)-E\left(c_{i}^{2} \mid \tau\right) E\left(c_{1} \mid \tau\right)-2 E\left(c_{i} \mid \tau\right) E\left(c_{i} c_{1} \mid \tau\right)\right. \\
& +\Sigma_{2}^{*} \beta x_{i}\left(E\left(c_{i} \mid \tau\right)\right)^{2}+\Sigma_{1}^{*} \beta x_{i} E\left(c_{i} c_{1} \mid \tau\right)+2 E\left(c_{1} \mid \tau\right)\left(E\left(c_{i} \mid \tau\right)\right)^{2} \\
& \left.-2 \Sigma_{1}^{*} \beta x_{i} E\left(c_{i} \mid \tau\right) E\left(c_{1} \mid \tau\right)\right] \\
& L_{124}=\frac{\partial L_{12}}{\partial \beta_{21}} \\
& =-\frac{\sum_{i=1} x_{i}^{2}}{\tau^{3}}\left[E\left(c_{i}^{2} c_{1} \mid \tau\right)-E\left(c_{i}^{2} \mid \tau\right) E\left(c_{1} \mid \tau\right)-2 E\left(c_{i} \mid \tau\right) E\left(c_{i} c_{1} \mid \tau\right)+\sum_{2}^{*} \beta x_{i}\left(E\left(c_{i} \mid \tau\right)\right)^{2}\right. \\
& +\Sigma_{1}^{*} \beta x_{i} E\left(c_{i} c_{1} \mid \tau\right)+2 E\left(c_{1} \mid \tau\right)\left(E\left(c_{i} \mid \tau\right)\right)^{2} \\
& \left.-2 \Sigma_{1}^{*} \beta x_{i} E\left(c_{i} \mid \tau\right) E\left(c_{1} \mid \tau\right)\right] \\
& L_{131}=L_{113} ; L_{132}=L_{123} \\
& L_{133}=\frac{\partial L_{13}}{\partial \beta_{20}}=-\frac{1}{\tau^{3}} \sum_{i=1}\left[E\left(c_{1}^{2} c_{i} \mid \tau\right)-2 \Sigma_{2}^{*} \beta x_{i} E\left(c_{i} c_{1} \mid \tau\right)+\left(\Sigma_{2}^{*} \beta x_{i}\right)^{2} E\left(c_{i} \mid \tau\right)\right. \\
& -E\left(c_{i} \mid \tau\right) E\left(c_{1}^{2} \mid \tau\right) \\
& \left.+E\left(c_{i} \mid \tau\right)\left(E\left(c_{i} \mid \tau\right)\right)^{2}\right] \\
& L_{134}=\frac{\partial L_{13}}{\partial \beta_{21}}=-\frac{\sum_{i=1} x_{i}}{\tau^{3}}\left[E\left(c_{1}^{2} c_{i} \mid \tau\right)-2 \Sigma_{2}^{*} \beta x_{i} E\left(c_{i} c_{1} \mid \tau\right)+\left(\Sigma_{2}^{*} \beta x_{i}\right)^{2} E\left(c_{i} \mid \tau\right)\right. \\
& -E\left(c_{i} \mid \tau\right) E\left(c_{1}^{2} \mid \tau\right) \\
& \left.+E\left(c_{i} \mid \tau\right)\left(E\left(c_{i} \mid \tau\right)\right)^{2}\right] \\
& L_{141}=L_{114} ; L_{142}=L_{124} ; L_{143}=L_{134}
\end{aligned}
$$




$$
\begin{aligned}
& L_{144}=\frac{\partial L_{14}}{\partial \beta_{21}}=-\frac{\sum_{i=1} x_{i}{ }^{2}}{\tau^{3}}\left[E\left(c_{1}^{2} c_{i} \mid \tau\right)-2 \Sigma_{2}^{*} \beta x_{i} E\left(c_{i} c_{1} \mid \tau\right)+\left(\Sigma_{2}^{*} \beta x_{i}\right)^{2} E\left(c_{i} \mid \tau\right)\right. \\
& -E\left(c_{i} \mid \tau\right) E\left(c_{1}^{2} \mid \tau\right) \\
& \left.+E\left(c_{i} \mid \tau\right)\left(E\left(c_{1} \mid \tau\right)\right)^{2}\right] \\
& L_{211}=L_{112} ; L_{212}=L_{122} ; L_{213}=L_{123} ; L_{214}=L_{124} ; L_{221}=L_{122} \\
& L_{222}=\frac{\partial L_{22}}{\partial \beta_{20}}=-\frac{\sum_{i=1} x_{i}^{3}}{\tau^{3}}\left[E\left(c_{i}^{3} \mid \tau\right)-2 \Sigma_{1}^{*} \beta x_{i} E\left(c_{i}^{2} \mid \tau\right)-E\left(c_{i} \mid \tau\right) E\left(c_{i}^{2} \mid \tau\right)\right. \\
& \left.+2\left(E\left(c_{i} \mid \tau\right)\right)^{3}\right] \\
& L_{223}=\frac{\partial L_{22}}{\partial \beta_{20}} \\
& =-\frac{\sum_{i=1} x_{i}^{2}}{\tau^{3}}\left[E\left(c_{i}^{2} c_{1} \mid \tau\right)-E\left(c_{i}^{2} \mid \tau\right) E\left(c_{1} \mid \tau\right)-2 E\left(c_{i} \mid \tau\right) E\left(c_{i} c_{1} \mid \tau\right)+\Sigma_{2}^{*} \beta x_{i}\left(E\left(c_{i} \mid \tau\right)\right)^{2}\right. \\
& +\Sigma_{1}^{*} \beta x_{i} E\left(c_{i} c_{1} \mid \tau\right)+2 E\left(c_{1} \mid \tau\right)\left(E\left(c_{i} \mid \tau\right)\right)^{2} \\
& \left.-2 \Sigma_{1}^{*} \beta x_{i} E\left(c_{i} \mid \tau\right) E\left(c_{1} \mid \tau\right)\right] \\
& L_{224}=\frac{\partial L_{22}}{\partial \beta_{21}}=-\frac{\sum_{i=1} x_{i}^{3}}{\tau^{3}}\left[E\left(c_{i}^{2} c_{1} \mid \tau\right)-E\left(c_{i}^{2} \mid \tau\right) E\left(c_{1} \mid \tau\right)-2 E\left(c_{i} \mid \tau\right) E\left(c_{i} c_{1} \mid \tau\right)\right. \\
& +\Sigma_{2}^{*} \beta x_{i}\left(E\left(c_{i} \mid \tau\right)\right)^{2}+\Sigma_{1}^{*} \beta x_{i} E\left(c_{i} c_{1} \mid \tau\right)+2 E\left(c_{1} \mid \tau\right)\left(E\left(c_{i} \mid \tau\right)\right)^{2} \\
& \left.-2 \Sigma_{1}^{*} \beta x_{i} E\left(c_{i} \mid \tau\right) E\left(c_{1} \mid \tau\right)\right] \\
& L_{231}=L_{123} ; L_{232}=L_{223} ; L_{233}=L_{322} ; L_{234}=L_{324} ; L_{311}=L_{113} \\
& L_{312}=L_{123} ; L_{313}=L_{133} ; L_{314}=L_{134} ; L_{321}=L_{123} ; L_{322}=L_{223} \\
& L_{323}=\frac{\partial L_{13}}{\partial \beta_{20}}=-\frac{\sum_{i=1} x_{i}}{\tau^{3}}\left[E\left(c_{1}^{2} c_{i} \mid \tau\right)-2 \Sigma_{2}^{*} \beta x_{i} E\left(c_{i} c_{1} \mid \tau\right)+\left(\Sigma_{2}^{*} \beta x_{i}\right)^{2} E\left(c_{i} \mid \tau\right)\right. \\
& \left.-E\left(c_{i} \mid \tau\right) E\left(c_{1}^{2} \mid \tau\right)+E\left(c_{i} \mid \tau\right)\left(E\left(c_{1} \mid \tau\right)\right)^{2}\right] \\
& L_{324}=\frac{\partial L_{32}}{\partial \beta_{21}}=-\frac{\sum_{i=1} x_{i}{ }^{3}}{\tau^{3}}\left[E\left(c_{1}^{2} c_{i} \mid \tau\right)-2 \Sigma_{2}^{*} \beta x_{i} E\left(c_{i} c_{1} \mid \tau\right)+\left(\Sigma_{2}^{*} \beta x_{i}\right)^{2} E\left(c_{i} \mid \tau\right)\right. \\
& \left.-E\left(c_{i} \mid \tau\right) E\left(c_{1}^{2} \mid \tau\right)+E\left(c_{i} \mid \tau\right)\left(E\left(c_{1} \mid \tau\right)\right)^{2}\right] \\
& L_{331}=L_{133} ; L_{332}=L_{232}=L_{323} \\
& L_{333}=-\frac{1}{\tau^{3}}\left[E\left(c_{1}^{3} \mid \tau\right)-E\left(c_{1} \mid \tau\right) E\left(c_{1}^{2} \mid \tau\right)\right] \\
& L_{334}=L_{433} ; L_{341}=L_{134} ; L_{342}=L_{234} ; L_{343}=L_{334}=L_{433} ; L_{344}=L_{342} \\
& L_{411}=L_{114} ; L_{412}=L_{124} ; L_{413}=L_{134} ; L_{414}=L_{114}=L_{411} ; L_{421}=L_{124} \\
& L_{422}=L_{224} ; L_{423}=L_{234}
\end{aligned}
$$




$$
\begin{aligned}
L_{424}=L_{244}= & \frac{\partial L_{42}}{\partial \beta_{21}} \\
& =-\frac{\sum_{i=1} x_{i}^{3}}{\tau^{3}}\left[E\left(c_{1}^{2} c_{i} \mid \tau\right)-2 \Sigma_{2}^{*} \beta x_{i} E\left(c_{i} c_{1} \mid \tau\right)+\left(\Sigma_{2}^{*} \beta x_{i}\right)^{2} E\left(c_{i} \mid \tau\right)\right. \\
& \left.-E\left(c_{i} \mid \tau\right) E\left(c_{1}^{2} \mid \tau\right)+E\left(c_{i} \mid \tau\right)\left(E\left(c_{1} \mid \tau\right)\right)^{2}\right]
\end{aligned}
$$

$$
\begin{aligned}
& L_{431}=L_{134} ; L_{432}=L_{234} ; L_{433}=L_{334} ; L_{434}=L_{344} ; L_{441}=L_{144} ; L_{442}=L_{244} ; L_{443} \\
& =L_{344} \\
& L_{444}=\frac{\partial L_{44}}{\partial \beta_{21}}=-\frac{\sum_{i=1} x_{i}^{3}}{\tau^{3}}\left[E\left(c_{1}^{3} \mid \tau\right)-2 \Sigma_{2}^{*} \beta x_{i} E\left(c_{1}^{2} \mid \tau\right)-E\left(c_{1} \mid \tau\right) E\left(c_{1}^{2} \mid \tau\right)\right. \\
& \left.+2\left(E\left(c_{1} \mid \tau\right)\right)^{3}\right]
\end{aligned}
$$

L تمثل المشتقة الثالثة من المشتقة الثانية ( المعادلة رقم (16) ) م بالنسبة الى 10

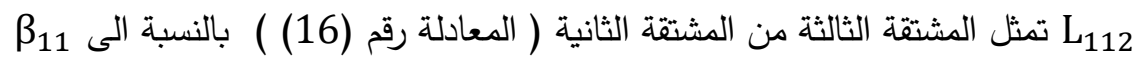

L المثل المشتقة الثالثة من المشتقة الثانية ( المعادلة رقم (16) ) ب بالنسبة الى

Lمثل المشتقة الثالثة من المشتقة الثانية ( المعادلة رقم (16) ) بالنسبة الى

ويمكن وضع هذه المشتقات في مصفوفة متماتلة وكما يلي :

$L_{16 * 4}^{(3)}=\left[\begin{array}{cccc}L_{111} & L_{112} & L_{113} & L_{114} \\ & L_{122} & L_{123} & L_{124} \\ \vdots & \ddots & L_{133} & L_{134} \\ & \ddots & \multicolumn{2}{c}{\vdots} \\ L_{441} & L_{442} & L_{443} & L_{444}\end{array}\right]$

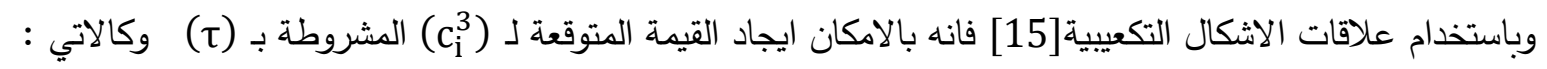
$E\left(c_{i}^{3}\right)=\Sigma_{1}^{*} \mathrm{M}_{3}\left(\Sigma_{1}^{* T} \otimes \Sigma_{1}^{* T}\right)+3 E c_{i}^{2} E c_{i}-2\left(E c_{i}\right)^{3}$

$M_{3}=E\left[\left(\left(y_{i}-m\right)\right)\left(y_{i}-m\right)^{\prime} \otimes\left(y_{i}-m\right)^{\prime} \mid \tau\right]$

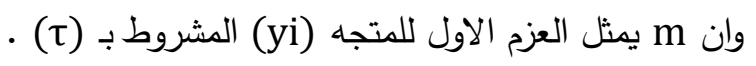

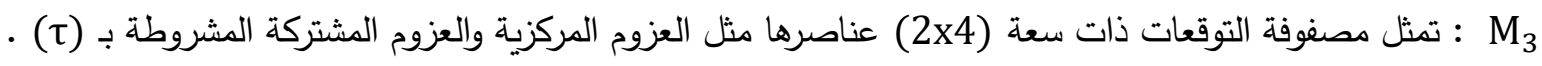
$\mathrm{M}_{3}=\left[\begin{array}{cccc}E y_{1}^{3} & E y_{1}^{2} y_{2} & E y_{1}^{2} y_{2} & E y_{1} y_{2}^{2} \\ E y_{1}^{2} y_{2} & E y_{1} y_{2}^{2} & E y_{1} y_{2}^{2} & E y_{2}^{3}\end{array}\right]$

$$
\begin{aligned}
& E\left(y_{i}^{r}\right)=E\left(y_{i}-E y_{i} \mid \tau\right)^{r} \mid \tau \\
& E\left(y_{i}^{r} y_{i}^{s}\right)=E\left(y_{i}-E y_{i} \mid \tau\right)^{r} E\left(y_{i}-E y_{i} \mid \tau\right)^{s} \quad r \neq s
\end{aligned}
$$


عندها ستكون تقديرات لندلي للمعلمات كما يلي :

$$
\begin{aligned}
& \hat{u}_{B}=u+\left(u_{1} a_{1}+u_{2} a_{2}+u_{3} a_{3}+u_{4} a_{4}+a_{5}\right) \\
& +\frac{1}{2}\left[A\left(u_{1} r_{11}+u_{2} r_{12}+u_{3} r_{13}+u_{4} r_{14}\right)+B\left(u_{1} r_{21}+u_{2} r_{22}+u_{3} r_{23}+u_{4} r_{24}\right)\right. \\
& +C\left(u_{1} r_{31}+u_{2} r_{32}+u_{3} r_{33}+u_{4} r_{34}\right) \\
& \left.+D\left(u_{1} r_{41}+u_{2} r_{42}+u_{3} r_{43}+u_{4} r_{44}\right)\right] \\
& a_{1}=\rho_{10} r_{11}+\rho_{11} r_{12}+\rho_{20} r_{13}+\rho_{21} r_{14}=0 \\
& a_{2}=\rho_{10} r_{21}+\rho_{11} r_{22}+\rho_{20} r_{23}+\rho_{21} r_{24}=0 \\
& a_{3}=\rho_{10} r_{31}+\rho_{11} r_{32}+\rho_{20} r_{33}+\rho_{21} r_{34}=0 \\
& a_{4}=\rho_{10} r_{41}+\rho_{11} r_{42}+\rho_{20} r_{43}+\rho_{21} r_{44}=0 \\
& a_{5}=\frac{1}{2}\left[u_{11} r_{11}+u_{22} r_{22}+u_{33} r_{33}+u_{44} r_{44}\right]=0 \\
& A=r_{11} L_{111}+2 r_{12} L_{121}+2 r_{13} L_{131}+2 r_{14} L_{141}+2 r_{23} L_{231}+2 r_{24} L_{241}+r_{22} L_{221}+r_{33} L_{331} \\
& +r_{44} L_{441} \\
& B=r_{11} L_{112}+2 r_{12} L_{122}+2 r_{13} L_{132}+2 r_{14} L_{142}+2 r_{23} L_{232}+2 r_{24} L_{242}+\quad r_{22} L_{222}+ \\
& r_{33} L_{332}+r_{44} L_{442} \\
& C=r_{11} L_{113}+2 r_{12} L_{123}+2 r_{13} L_{133}+2 r_{14} L_{143}+2 r_{23} L_{233}+2 r_{24} L_{243}+r_{22} L_{223}+r_{33} L_{333} \\
& +r_{44} L_{443} \\
& D=r_{11} L_{114}+2 r_{12} L_{124}+2 r_{13} L_{134}+2 r_{14} L_{144}+2 r_{23} L_{234}+2 r_{24} L_{244}+r_{22} L_{224} \\
& +r_{33} L_{334}+r_{44} L_{444}
\end{aligned}
$$

: مقدر بيز للمعلمة 10

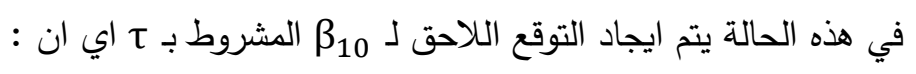

$u\left(\beta_{10} \mid \tau\right)=\beta_{10}$

$$
u_{1}=\frac{\partial u}{\partial \beta_{10}}=1 ; u_{2}=\frac{\partial u}{\partial \beta_{11}}=0 ; u_{3}=\frac{\partial u}{\partial \beta_{20}}=0 ; u_{4}=\frac{\partial u}{\partial \beta_{21}}=0
$$$$
u_{11}=\frac{\partial^{2} u}{\partial \beta_{0}^{2}}=0
$$$$
u_{12}=u_{13}=u_{21}=u_{22}=u_{23}=u_{31}=u_{32}=u_{33}=0
$$$$
\frac{\partial \rho_{10}}{\partial \beta_{10}}=\frac{\partial \rho_{20}}{\partial \beta_{11}}=\frac{\partial \rho_{20}}{\partial \beta_{20}}=\frac{\partial \rho_{21}}{\partial \beta_{21}}=0 \rightarrow \rho_{10}=\rho_{11}=\rho_{20}=\rho_{21}
$$$$
\hat{\beta}_{10 B \mid \tau}=\hat{\beta}_{10}+\left.\frac{1}{2}\left[A r_{11}+B r_{21}+C r_{31}+D r_{41}\right]\right|_{\beta_{10}=\hat{\beta}_{10}}
$$ 


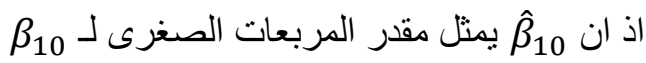

$$
\begin{aligned}
& \text { اما مقدر بيز غير الثرطي لـ }
\end{aligned}
$$

$\hat{\beta}_{10 B}=E E\left(\hat{\beta}_{10 \beta} \mid\right.$ data,$\left.\tau\right)=E \int_{0}^{\infty}\left(\hat{\beta}_{10 B} \mid \tau\right) f(\tau) d \tau$

: مقدر بيز للمعلمة

$u\left(\beta_{11} \mid \tau\right)=\beta_{11}$

$u_{1}=\frac{\partial u}{\partial \beta_{10}}=0 ; u_{2}=\frac{\partial u}{\partial \beta_{11}}=1 ; u_{3}=\frac{\partial u}{\beta_{20}}=0 ; u_{4}=\frac{\partial u}{\beta_{21}}=0$

$u_{22}=\frac{\partial^{2} u}{\partial \beta_{10}^{2}}=0$

$u_{11}=u_{12}=u_{13}=u_{21}=u_{23}=u_{31}=u_{32}=u_{33}=0$

$E\left(\hat{\beta}_{11 B} \mid\right.$ data,$\left.\tau\right)=\hat{u}_{B \mid \tau}=\hat{\beta}_{11}+\frac{1}{2}\left[A r_{12}+B r_{22}+C r_{32}\right]$

$\hat{\beta}_{11 B}=E E\left(\hat{\beta}_{11 B} \mid\right.$ data,$\left.\tau\right)=E \int_{0}^{\infty}\left(\hat{\beta}_{11 B} \mid \tau\right) f(\tau) d \tau$

:

$u\left(\beta_{20} \mid \tau\right)=\beta_{20}$

$u_{1}=\frac{\partial u}{\partial \beta_{10}}=0 ; u_{2}=\frac{\partial u}{\partial \beta_{11}}=0 ; u_{3}=\frac{\partial u}{\beta_{20}}=1 ; u_{4}=\frac{\partial u}{\beta_{21}}=0$

$u_{33}=\frac{\partial^{2} u}{\partial \beta_{20}^{2}}=0$

$u_{11}=u_{12}=u_{13}=u_{21}=u_{23}=u_{31}=u_{32}=u_{33}=0$

$E\left(\hat{\beta}_{20 B} \mid\right.$ data,$\left.\tau\right)=\hat{u}_{B \mid \tau}=\hat{\beta}_{20}+\frac{1}{2}\left[A r_{13}+B r_{23}+C r_{33}+D r_{43}\right]$

$\hat{\beta}_{20 B}=E E\left(\hat{\beta}_{20 B} \mid\right.$ data $\left.\tau\right)=E \int_{0}^{\infty}\left(\hat{\beta}_{20 B} \mid \tau\right) f(\tau) d \tau$

: يتم ايجاد التوقع اللاحق لـ

$u\left(\beta_{21} \mid \tau\right)=\beta_{21}$

$u_{1}=\frac{\partial u}{\partial \beta_{10}}=0 ; u_{2}=\frac{\partial u}{\partial \beta_{11}}=0 ; u_{3}=\frac{\partial u}{\beta_{20}}=0 ; u_{4}=\frac{\partial u}{\beta_{21}}=1$

$u_{44}=\frac{\partial^{2} u}{\partial \beta_{20}^{2}}=0$ 


$$
\begin{aligned}
& u_{11}=u_{12}=u_{13}=u_{21}=u_{23}=u_{31}=u_{32}=u_{33}=0 \\
& \text { وعليه فان مقدر بيز لـ } \\
& E\left(\hat{\beta}_{21 B} \mid \text { data }, \tau\right)=\hat{u}_{B \mid \tau}=\hat{\beta}_{21}+\frac{1}{2}\left[A r_{14}+B r_{24}+C r_{34}+D r_{44}\right] \\
& \hat{\beta}_{21 B}=E E\left(\hat{\beta}_{21 B} \mid \text { data }, \tau\right)=E \int_{0}^{\infty}\left(\hat{\beta}_{21 B} \mid \tau\right) f(\tau) d \tau
\end{aligned}
$$

$$
\text { هناك عدة استتتاجات تم التوصل اليها وكما يلي : }
$$

يواجه تقريب لندلي صعوبة وجود الدالة التجميعية في التوزيع اللاحق ، اذ ان هذه الدالة لا يمكن معاملتها كثابت لأنها تعتمد على معلمات التوزيع الاحتمالي ، وهذه المعلمات هي متغيرات عشوائية بأسلوب بيز ومن الصعب جدا ايجاد مقدرات بيز الهامشية ، بينما بالتقريب المستخدم تمكنا من ايجاد المقدرات الهامشية من دون الحاجة الى ايجاد التوزيعات اللاحقة الهامشية. ان جميع مقدرات معلمات الانحدار اعتمدت على عزوم متغيرات الاستجابة لغاية الرتبة الثالثة .

1- Fernandez.C, Steel..J (1997). Center for economic research .

2- Yan-Hui , S (2003). School of Mathematics, Papers of Xiao-Zhang Scholars .

3- Alathari, M.F., Al-sarraf ,J.Z. (2008) . Al-Manarah,vol. 14, No. 3.

4- Massuia,M. B., Celso R. B., Larissa A. M. ,and Víctor H. L. (2007). Departamento de Estatística, Universidade Estadual de Cam pinas, Brazil .

5- Ho,H J. , Lin,T-I , Chen.H. , Wang,Wan-Lun (2012) . journal of statistical planning and inference 142 (2012) 25 - 40 , www.elsevier.com/locate/jspi

6- Hormuz, A. H. (1999. Dar Al Kutub Books and Publishing.

7- Nadarajah,saralees, Kots,Samuel,(2008) . Acta Applemeth (2008)102:99-118 , DOI $10.1007 / \mathrm{s}$ 10440-008-9212-8.

8- Inmaculada B. Aban,M.M. , Anna K. P ( 2006) : Journal of the American Statistical Association ; Vol. 101, No. 473 , March 2006. 
9- Nadarajah,saralees , Kots,Samuel,(2005) . Canadian applied, mathematics quarterly, Volume 13, Number 1, Spring 2005

10- Aljboory, Shlal Habeeb , Abd,Salah Hamza (2000) . Baghdad, Dar Al Kutub for Printing and Publishing.

11- Kelly,B.C(2007). The Astrophysical Journal ,665 : 1489-1506,2007 August 20 ; Printed in U.S.A .

12- Griffiths,W.(2001) . Dep. Of economic , university of Melbourne.

13- Mohamad ,Raya Salem ,Dham,Watheq Nathem : (2013). Iraqi Journal of Statistical Sciences, (24) 2013, pp. 51-69.

14- Al-kanani.I. H. ,Samier.F,O ( 2010 ) . Journal of college of education , No 6, 2010 , University of Baghdad, Iraq .

15- Peterson,K. B.and, Pedersen,M. S. (2012) .

http : // matrixcookbook .com 